Check for updates

Cite this: RSC Adv., 2017, 7, 49613

Received 22nd August 2017 Accepted 5th October 2017

DOI: $10.1039 / \mathrm{c} 7 \mathrm{ra09250d}$

rsc.li/rsc-advances

\section{Effects of $\mathrm{Ga}_{x} \mathrm{Zn}_{1-x} \mathrm{O}$ nanorods on the photoelectric properties of $\mathrm{n}-\mathrm{ZnO}$ nanorods/p- GaN heterojunction light-emitting diodes}

\author{
Rui Li, (D) ab Chunyan Yu, ${ }^{\text {ab }}$ Hailiang Dong, ${ }^{\text {ab }}$ Wei Jia, ${ }^{\text {ab }}$ Tianbao Li, ${ }^{\text {ab }}$ \\ Zhuxia Zhang (DD ab and Bingshe $\mathrm{Xu}^{\mathrm{ab}}$
}

A Ga-doped $\mathrm{ZnO}$ nanorod array has been synthesized on a $\mathrm{p}$ $\mathrm{GaN} / \mathrm{Al}_{2} \mathrm{O}_{3}$ substrate by a hydrothermal method at low temperature. The structures and morphologies of the samples were measured by XRD and FE-SEM. The results show that the Ga-doped ZnO nanorods have excellent crystallinity and good epitaxial relationships with the substrate. With increasing Ga doping, the ZnO nanorods will grow along the [001] direction rapidly resulting in a decreasing average diameter. At the same time, the incorporation of Ga also significantly affects the optical and electrical properties of the $n-\mathrm{ZnO}$ nanorods/p-GaN heterojunction light-emitting diode. From the photoluminescence spectrum, it was found that Ga doping can be effectively regulated by the UV and visible emission peak intensity ratio of the $\mathrm{ZnO}$ nanorods. The $I-V$ characteristics curve indicates that the $\mathrm{n}-\mathrm{ZnO}$ nanorods/p-GaN heterojunction light-emitting diode will have better conductivity with increasing Ga doping concentration. Finally, the heterojunction light-emitting diode achieves green light emission under forward bias.

\section{Introduction}

One-dimensional nanostructures such as nanorods (NRs) and nanowires (NWs) have been extensively studied, ${ }^{1-3}$ since their high crystallinity can provide an effective electron conduction path, ${ }^{4}$ and the vertical arrangement of NRs/NWs can also reduce the light of lateral scattering and enhance the light extraction efficiency, ${ }^{5,6}$ which makes it better than traditional planar $\mathrm{p}-\mathrm{n}$ junction light-emitting diodes (LEDs).

$\mathrm{ZnO}$ has wide application prospects in the fields of LEDs, LDs and solar cells because of its wide direct band gap $(3.37 \mathrm{eV})$ and high exciton binding energy $(60 \mathrm{meV})$ at room

${ }^{a}$ Key Laboratory of Interface Science and Engineering in Advanced Materials, Ministry of Education, Taiyuan University of Technology, Taiyuan 030024, China. E-mail: yuchunyan75@163.com

${ }^{b}$ Research Center of Advanced Materials Science and Technology, Taiyuan University of Technology, Taiyuan 030024, China temperature. ${ }^{--9}$ For the application of $\mathrm{ZnO}$-based optoelectronic devices, it is necessary to fabricate both $\mathrm{n}-\mathrm{ZnO}$ and $\mathrm{p}-\mathrm{ZnO}$. In recent years, although certain progress has been made in fabricating $\mathrm{p}$-ZnO and even ZnO-based $\mathrm{p}-\mathrm{n}$ homojunction LEDs, it is still difficult to prepare stable and reproducible highquality p-type $\mathrm{ZnO}$, which hinders the development of $\mathrm{ZnO} \mathrm{p}-\mathrm{n}$ homojunction LEDs. ${ }^{10,11}$ So far, many efforts have been devoted to developing ZnO-based $\mathrm{p}-\mathrm{n}$ heterojunction LEDs with $\mathrm{ZnO}$ as the n-type layer, combined with other p-type layers, such as $\mathrm{p}$ $\mathrm{Si},{ }^{12} \mathrm{p}-\mathrm{GaN},{ }^{13} \mathrm{p}-\mathrm{AlGaN},{ }^{14,15}$ and $\mathrm{p}$-GaAs. ${ }^{16}$ Among them, it is important to explore a material that matches GaN, because both GaN and $\mathrm{ZnO}$ have the same crystal structure (hexagonal wurtzite), with a smaller lattice mismatch (1.8\%) and with a similar room temperature wide band gap $(3.4 \mathrm{eV}){ }^{17,18}$

However, since the energy barrier of the $\mathrm{n}-\mathrm{ZnO} / \mathrm{p}-\mathrm{GaN}$ junction interface leads to the low injection efficiency of carriers, the emission efficiency and output power of the heterojunction LED cannot meet the requirements of high performance devices. ${ }^{19}$ This problem can be improved by adding metal elements, such as $\mathrm{Al}, \mathrm{Ga}$, In and so on because these elements could form a doping level in the conduction band.

Ga doping can achieve smaller lattice distortions in $\mathrm{Ga}_{x^{-}}$ $\mathrm{Zn}_{1-x} \mathrm{O}$ than $\mathrm{Al}$ or In doping under high doping concentrations because the bond length of $\mathrm{Ga}-\mathrm{O}(1.92 \AA)$ is closer to the bond length of $\mathrm{Zn}-\mathrm{O}(1.97 \AA)$ than that of Al-O (2.70 $)$ or In-O (2.1 A). ${ }^{20}$ Zehao et al. prepared Ga doped ZnO NR photodetectors with a smaller dark current and faster response speed by a low temperature hydrothermal method. ${ }^{21} \mathrm{He}$ et al. synthesized individual heavily Ga-doped $\mathrm{ZnO}$ microwires via chemical vapor deposition methods, and bright, stable, and near-infrared lightemission from electrically biased individual $\mathrm{Ga}$ doped $\mathrm{ZnO}$ microwires was achieved. ${ }^{22}$ Studies have shown that Ga doping can significantly affect the growth behavior as well as the optical and electrical properties and defect states of ZnO NRs. ${ }^{23}$ Although there are a lot of reports on this issue, a disagreement still exists because the growth of NRs is sensitive to the doping concentration, reaction time, temperature and so on. ${ }^{24,25}$ In addition, little attention has been paid to the effect of Ga doping 
on the photoelectric properties of $\mathrm{n}-\mathrm{ZnO} \mathrm{NRs} / \mathrm{p}-\mathrm{GaN}$ heterojunction LEDs.

In this work, a Ga-doped ZnO NR array has been synthesized on a p-GaN/ $\mathrm{Al}_{2} \mathrm{O}_{3}$ substrate. The effects of Ga doping concentration (0-3\%) on the growth behaviour of ZnO NRs have been investigated and the photoelectric properties of the $\mathrm{Ga}_{x} \mathrm{Zn}_{1-x} \mathrm{O}$ NRs/p-GaN heterojunction LED are discussed.

\section{Experimental}

Ga-doped ZnO NRs were synthesized on p-GaN/ $/ \mathrm{Al}_{2} \mathrm{O}_{3}$ (001) substrates by a low temperature hydrothermal growth process. The Mg-doped p-GaN film was grown on c- $\mathrm{Al}_{2} \mathrm{O}_{3}$ substrates using metal organic chemical vapor deposition. To grow more regular $\mathrm{ZnO}$ NRs on the p-GaN substrates, a ZnO seed layer was deposited on the substrate. ${ }^{26}$ The seed layer was then immersed in an aqueous solution containing a mixture of $0.075 \mathrm{M}$ $\mathrm{Zn}\left(\mathrm{NO}_{3}\right)_{2} \cdot 6 \mathrm{H}_{2} \mathrm{O}$ and equivalent molar hexamethylenetetramine $\left(\mathrm{HMT}, \mathrm{C}_{6} \mathrm{H}_{12} \mathrm{~N}_{4}\right.$ ) at approximately $95{ }^{\circ} \mathrm{C}$ for $4.5 \mathrm{~h}$. To obtain the Ga-doped samples, $\mathrm{Ga}\left(\mathrm{NO}_{3}\right)_{3} \cdot x \mathrm{H}_{2} \mathrm{O}$ was added to the aqueous growth solution with nominal concentrations of 1 to $3 \mathrm{~mol} \%$. Hereafter, undoped $\mathrm{ZnO}$ and Ga-doped $\mathrm{ZnO}$ are denoted as UZO and GZO, respectively. To investigate the electrical properties of the heterojunction, Au electrodes were deposited onto the $\mathrm{ZnO}$ and GaN films by thermal evaporation. We first filled the gap of the nanorods with photoresist before depositing the electrodes to ensure that the electrode would have direct contact with the top of the nanorod. A schematic diagram of the structure of the heterojunction LED is shown in Fig. 1.

The crystal structure of the sample was analyzed using a Japanese Y-2000 type X-ray diffractometer $(\mathrm{Cu} \mathrm{K} \alpha$ ray, $\lambda=$ $0.15418 \mathrm{~nm}$ ), the morphology of the samples was analyzed by JEOL JSM6700F field emission scanning electron microscopy (FE-SEM), and the optical properties of the samples were analyzed by a Nanometrics Corporation NAN-RPM2000 Photoluminescence (PL) spectrometer (excitation wavelength: 266 $\mathrm{nm})$. The electrical properties of the samples were tested with the FS-TL series LED test equipment.

\section{Results and discussion}

The influence of Ga doping on the structural properties of $n$ ZnO NRs was investigated by XRD and FE-SEM. Fig. 2 shows

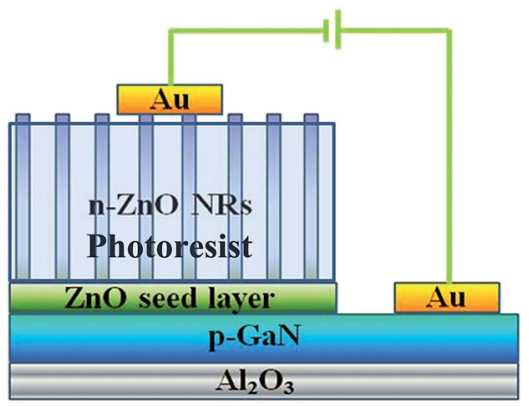

Fig. 1 Schematic diagram of the structure of the heterojunction LED.

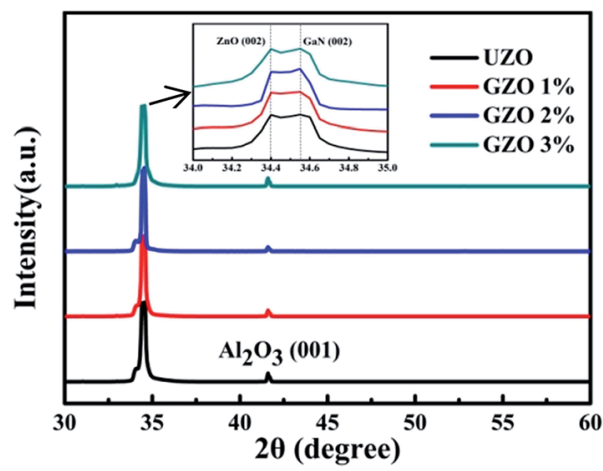

Fig. 2 XRD patterns of the $\mathrm{ZnO}$ NR arrays with different Ga doping concentrations. The inset shows the enlarged view of the (002) peak.

the XRD patterns of the $\mathrm{n}-\mathrm{ZnO} \mathrm{NRs} / \mathrm{p}-\mathrm{GaN}$ arrays with respect to the Ga doping concentration and its inset shows the enlarged view of the (002) peak. Strong diffraction peaks at $34.4^{\circ}$ and $34.5^{\circ}$ can be assigned to $\mathrm{ZnO}(002)$ and $\mathrm{GaN}(002)$ corresponding to JCPDS 36-1451 and JCPDS 50-0792, respectively. This indicates that all samples exhibit a wurtzite hexagonal structure regardless of the growth conditions. Beyond that, the (002) diffraction peaks of all samples are sharp and the NRs have a [001] growth direction, indicating that the ZnO NRs have excellent crystallinity and a good epitaxial relationship with the p-GaN epitaxial film. No additional peak related either to Ga or its oxides was detected, which indicates that the Ga atoms have been solid-solved into the interior of the $\mathrm{ZnO}$ lattice. As can be seen in the enlarged view of the (002) peaks, the $\mathrm{ZnO}(002)$ peak position does not show obvious displacement with the increase of the Ga doping concentration, indicating that Ga doping does not cause lattice mismatch.

Fig. 3(a)-(d) exhibit the plan-view SEM images of the morphologies of the UZO and GZO NRs, and Table 1 presents the distribution of the different elements ( $\mathrm{Zn}, \mathrm{O}$ and $\mathrm{Ga}$ ) in the UZO and GZO NRs detected by EDS. For all samples, the hexagonal-shaped NRs were vertically grown on the p-GaN film, which is consistent with the XRD results. The average diameter and the number of NRs per unit estimated from the corresponding SEM images are plotted in Fig. 3(e). It can be seen intuitively that the average diameter and the density both
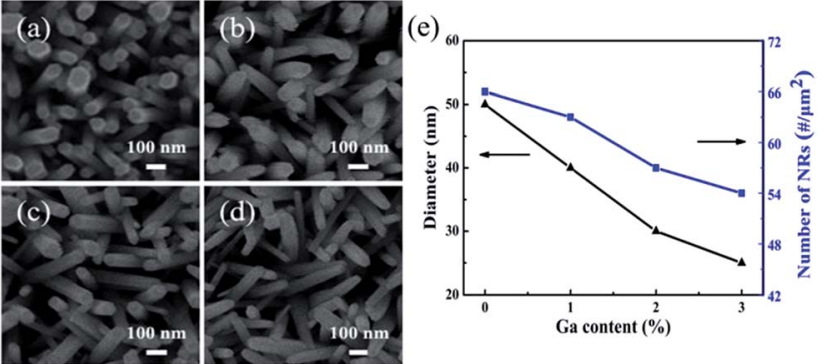

Fig. 3 Plan-view FE-SEM images of the GZO NRs with different Ga concentrations: (a) $0 \%$, (b) $1 \%$, (c) $2 \%$, and (d) $3 \%$, and (e) variations of the average diameter and density. 
Table 1 The distribution of elements $\mathrm{Zn}$ and Ga detected by EDS

\begin{tabular}{lllll}
\hline & UZO & GZO 1\% & GZO 2\% & GZO 3\% \\
\hline Zn atomic percent & $55.3 \%$ & $55.1 \%$ & $53.4 \%$ & $48.2 \%$ \\
Ga atomic percent & $0 \%$ & $0.2 \%$ & $0.4 \%$ & $0.7 \%$ \\
$\mathrm{Ga} /(\mathrm{Ga}+\mathrm{Zn})$ ratio & $0 \%$ & $0.4 \%$ & $0.7 \%$ & $1.4 \%$ \\
\hline
\end{tabular}

decrease as the Ga concentration increases. In addition, the uniformity of the NRs deteriorates with increasing doping concentration. It has been reported that the ZnO NRs will grow along the [001] direction rapidly with Ga doping, thus there is a decrease in the average diameter. ${ }^{27}$ Moreover, the incorporation of Ga ions inhibits the formation of the $\mathrm{ZnO}$ nuclei, ${ }^{21}$ so the density of the NRs decreases with increasing Ga doping concentration. These results demonstrate that the growth behavior of the ZnO NRs is significantly affected by Ga doping. The comparison of the EDS data with different doping concentration shows that with an increase in doping concentration, the ratio of $\mathrm{Ga}$ to $\mathrm{Zn}$ in the $\mathrm{ZnO}$ NRs increases, indicating that $\mathrm{Ga}$ atoms have been incorporated into the $\mathrm{ZnO}$ NRs effectively.

Fig. 4 depicts the photoluminescence spectra of p-GaN (a) and $n-Z n O$ NRs/p-GaN with different Ga doping concentrations (b). p-GaN has a relatively sharp UV emission peak at $360 \mathrm{~nm}$. Fig. 4(b) shows two emission peaks for all samples: a strong UV emission peak located at $358 \mathrm{~nm}$ and an almost negligible visible emission peak at about $620 \mathrm{~nm}$. The UV emission peak is attributed to the recombination of free excitons through a near band edge exciton-exciton collision process, and the visible emission peak is associated with the electron-hole recombination at deep levels caused by intrinsic defects such as oxygen vacancies and/or zinc interstitials. ${ }^{21}$ In this experiment, the low intensity of the defect emission peak indicates that the prepared ZnO NRs have good crystallinity and excellent optical efficiency. A similar result was obtained by Rogers et al. ${ }^{28}$ They reported a $\mathrm{ZnO} / \mathrm{GaN} \mathrm{p}-\mathrm{n}$ heterojunction LED using a pulsed laser, and the PL showed a main peak at $375 \mathrm{~nm}$ and a negligibly low green emission. With increasing Ga doping concentration, the intensity of the UV peak of the ZnO NRs first increases and then decreases, reaching a maximum peak in the GZO 1\% sample. The illustration in Fig. 4(b) is a visible emission peak magnified $50 \times$, and its intensity decreases with the increase of Ga doping. It is generally believed that the ratio of
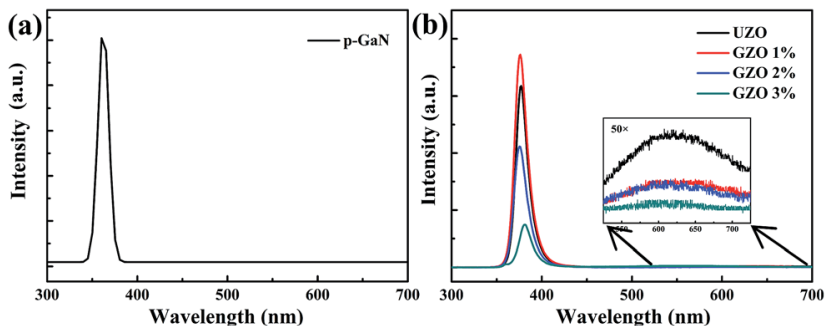

Fig. 4 Photoluminescence spectra of the $\mathrm{p}-\mathrm{GaN}(\mathrm{a})$ and the $\mathrm{n}-\mathrm{ZnO}$ NRs with different Ga doping concentrations (b). the UV peak to the visible emission peak is related to the crystal quality of the sample: the larger the ratio, the higher the crystal quality. Therefore, when the Ga doping concentration is $1 \%$, the sample has the best crystalline quality, which is in agreement with the XRD results. When the Ga doping content increases further, the UV peak intensity reduces because of the introduction of more nonradiative recombination centers. Therefore, the incorporation of $\mathrm{Ga}$ can affect the crystal quality of $\mathrm{ZnO}$. Beyond that, there was a small redshift of the peak in the UV emission, which can be ascribed to the Ga-doping induced band gap renormalization effect. ${ }^{29}$

Fig. 5 exhibits the $I-V$ characteristics of the ZnO NRs/p-GaN heterojunction with different Ga doping concentrations. The inset shows the $I-V$ characteristics of $\mathrm{Au} / \mathrm{GaN}$ and $\mathrm{Au} / \mathrm{ZnO}$, indicating that there is good ohmic contact between the electrode and the material. p-GaN acts not only as a ZnO NR buffer layer, but also as a p-type hole injection layer. And the hole concentration of the $\mathrm{p}-\mathrm{GaN}$ is about $4.0 \times 10^{17}$. The curves of all samples show rectification characteristics. The current increases with increasing voltage and at the same forward bias, the higher the Ga doping concentration, the greater the current of the heterojunction. This phenomenon indicates that the electrical conductivity of the ZnO NRs increases with the increase of the Ga content. This is because the increasing Ga doping concentration results in the number of carriers (electrons) increasing in the $\mathrm{ZnO} \mathrm{NRs}$, thus reducing the resistance of the heterojunctions.

It is observed that the heterojunction LED luminescence is on the ZnO side when a forward bias is applied. Fig. 6(a) shows the electroluminescence spectra of the 1\% GZO nanorods/pGaN heterojunction when the bias voltage is $30 \mathrm{~V}$ (a) and the emission peak is centered at $520 \mathrm{~nm}$ at a reverse bias voltage of 30 V. Fig. 6(b) is the CIE 1931 color coordinate measurement for the heterojunction LEDs at $30 \mathrm{~V}$ when the Ga doping content is $1 \%$, and the chromaticity coordinate is $(0.3228,0.4591)$. As the image illustrates in the inset of Fig. 6(b), the EL emission is clearly seen by the naked eye. Similar results for the light emission of an n-ZnO NRs/p-GaN heterojunction LED have been reported. Fu et al. demonstrated a $\mathrm{ZnO}$ NRs/p-GaN heterojunction LED, and observed green emission centered at $519 \mathrm{~nm}$ at a reverse bias voltage of $3.5 \mathrm{~V}$ that originated from oxygen vacancies near the heterojunction interface. ${ }^{30}$ The origin of the EL emissions can be determined from the energy band diagram

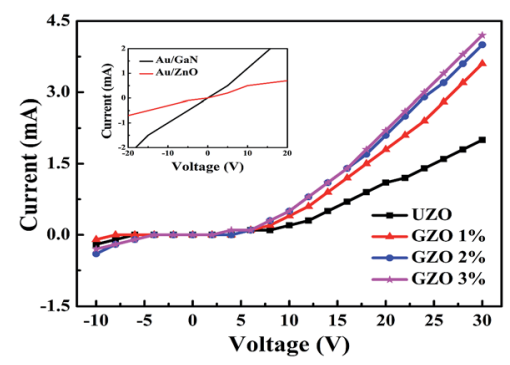

Fig. $5 \quad I-V$ characteristics of the $\mathrm{n}-\mathrm{ZnO} \mathrm{NRs} / \mathrm{p}-\mathrm{GaN}$ heterojunction LED with different $G$ a doping concentrations. The inset shows the I-V characteristics of $\mathrm{Au} / \mathrm{ZnO}$ and $\mathrm{Au} / \mathrm{GaN}$. 

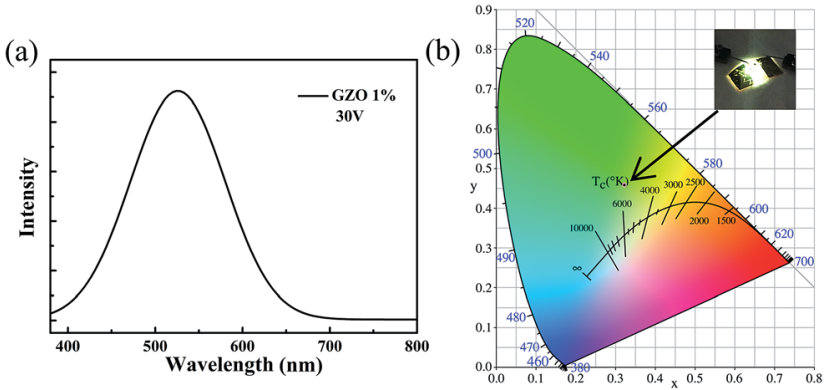

Fig. 6 Electroluminescence spectra of the 1\% GZO nanorods/p-GaN heterojunction when the bias voltage is $30 \mathrm{~V}(\mathrm{a})$ and the chromaticity coordinates of the light emission from the LED operated at $30 \mathrm{~V}$ (b). The inset of (b) shows the image of the device at the corresponding voltage.
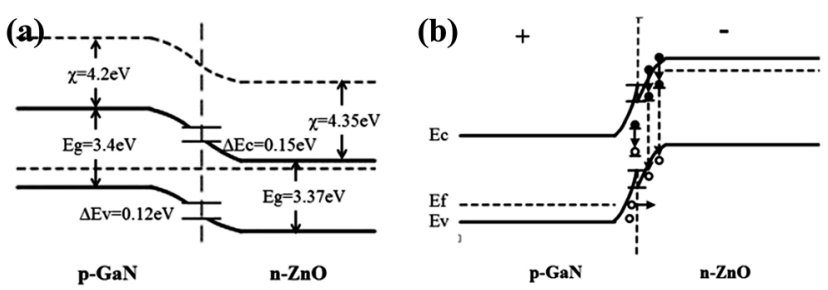

Fig. 7 The energy band diagram of the $n-Z n O ~ N R s / p-G a N$ heterojunction without applying a voltage (a) and applying forward bias (b).

shown in Fig. 7, combined with the photoluminescence spectra of the p-GaN and the n-ZnO NRs/p-GaN with different Ga doping concentrations as shown in Fig. 4.

The electron affinities of $\mathrm{ZnO}\left(\chi_{\mathrm{ZnO}}\right)$ and $\mathrm{GaN}\left(\chi_{\mathrm{GaN}}\right)$ are 4.35 and $4.2 \mathrm{eV}$. The conduction band offset is $\Delta E_{\mathrm{c}}=\chi_{\mathrm{ZnO}}-\chi_{\mathrm{GaN}}=$ $0.15 \mathrm{eV}$, and the valence band offset is $\Delta E_{\mathrm{v}}=E_{\mathrm{g} \mathrm{ZnO}}+\Delta E_{\mathrm{c}}-E_{\mathrm{g}}$ $\mathrm{GaN}=0.12 \mathrm{eV}$, according to the Anderson model. Although the electron mobility is greater than that of the hole, the holes in pGaN are more likely to be implanted into $\mathrm{ZnO}$ due to the fact that the barrier of the holes is smaller than that of the electrons and the depth of the depletion layer required for the holes is much smaller than that of the electrons. When a forward bias is applied, the electrons injected into the $\mathrm{ZnO}$ conduction band are easily occupied by the low-energy electrons associated with the defects, and are recombined with the holes injected from $\mathrm{GaN}$ into the $\mathrm{ZnO}$ valence band and into $\mathrm{ZnO}$ intrinsic holes.

\section{Conclusions}

A Ga $\mathrm{Zn}_{1-x} \mathrm{O}$ NRs/p-GaN heterojunction LED has been fabricated. Test results show that Ga doping can significantly affect the growth behavior of ZnO NRs and the photoelectric properties of the n-ZnO NRs/p-GaN heterojunction LED. When the Ga doping concentration is $1 \%$, the crystal quality and optical properties of the sample are optimal. Heterojunction LEDs have better conductivity with the increase of Ga doping concentration. A strong green emission is obtained when the Ga doping concentration is $1 \%$. The origin of the EL emission is supposed to be attributed to the defects in the heterojunction combined with an energy band structure diagram. This method provides a meaningful approach for the preparation of ZnO-based optoelectronic devices achieving excellent performance.

\section{Conflicts of interest}

There are no conflicts to declare.

\section{Acknowledgements}

This work is financially supported by the National Natural Science Foundation of China (Grant no. 21471111, 61475110, 61404089, 61504090 and 61604104), the Natural Science Foundation of Shanxi Province (Grant no. 2014011016-6 and 2015011034), the Basic Research Projects of Shanxi Province (Grant no. 2015021103) and the Shanxi Provincial Key Innovative Research Team in Science and Technology (Grant no. 201605D131045-10).

\section{References}

1 Z. Liang, X. Cai, S. Tan, et al., Phys. Chem. Chem. Phys., 2012, 14, 16111-16114.

2 X. W. Sun, J. Z. Huang, J. X. Wang, et al., Nano Lett., 2008, 8, 1219-1223.

3 Y. Zhang, D. Q. Fang, S. L. Zhang, et al., Phys. Chem. Chem. Phys., 2016, 18, 3097-3102.

4 Y. J. Lee, Z. P. Yang, F. Y. Lo, et al., Apl Materials, 2014, 2(5), 42.

5 H. Jeong, D. J. Park, H. S. Lee, et al., Nanoscale, 2014, 6, 43714378.

6 B. U. Ye, B. J. Kim, Y. H. Song, et al., Adv. Funct. Mater., 2012, 22, 632-639.

7 Ü. Özgür, Y. I. Alivov, C. Liu, et al., J. Appl. Phys., 2005, 98, 11.

8 C. Bayram, F. Hosseini Teherani, D. J. Rogers, et al., Appl. Phys. Lett., 2008, 93, 459.

9 X. N. Wang, Y. Wang, Z. X. Mei, et al., Appl. Phys. Lett., 2007, 90, 2720.

10 C. Klingshirn, ChemPhysChem, 2007, 8(6), 782.

11 D. C. Look, B. Claflin, Y. I. Alivov, et al., Phys. Status Solidi, 2004, 201, 2203-2212.

12 G. Xiong, J. Wilkinson, S. Tuzemen, et al., Proc. SPIE, 2011, 4644, 256-262.

13 Y. I. Alivov, J. E. Van Nostrand, D. C. Look, et al., Appl. Phys. Lett., 2003, 83, 2943-2945.

14 Q. X. Yu, B. Xu, Q. H. Wu, et al., Appl. Phys. Lett., 2003, 83, 4713-4715.

15 Y. I. Alivov, E. V. Kalinina, A. E. Cherenkov, et al., Appl. Phys. Lett., 2003, 83, 4719-4721.

16 S. E. Nikitin, Y. A. Nikolaev, V. Y. Rud, et al., Semiconductors, 2004, 38, 393-396.

17 T. P. Yang, H. C. Zhu, J. M. Bian, et al., Mater. Res. Bull., 2008, 43, 3614-3620.

18 S. Fiatvarol, D. Sahin, M. Kompitsas, et al., RSC Adv., 2014, 4, 13593-13600.

19 S. K. Hong, T. Hanada, H. Makino, et al., Appl. Phys. Lett., 2001, 78, 3349-3351. 
20 S. J. Young, C. L. Chiou, Y. H. Liu, et al., Inventions, 2016, 1, 3. 21 S. Zehao, Z. Hai, Y. Cong, et al., Mater. Sci. Semicond. Process., 2017, 64, 101-108.

22 G. H. He, M. M. Jiang, L. Dong, et al., J. Mater. Chem. C, 2017, 5, 2542-2551.

23 G. C. Park, S. M. Hwang, J. H. Lim, et al., Nanoscale, 2014, 6, 1840-1847.

24 G. Pineda-Hernández, A. Escobedo-Morales, U. Pal, et al., Mater. Chem. Phys., 2012, 135, 810-817.

25 J. Guo, J. Zheng, X. Song, et al., Mater. Lett., 2013, 97, 34-36.
26 T. A. N. Peiris, H. Alessa, J. S. Sagu, et al., J. Nanopart. Res., 2013, 15, 2115.

27 J. Joo, B. Y. Chow, M. Prakash, et al., Nat. Mater., 2011, 10, 596.

28 D. J. Rogers, F. H. Teherani, A. Yasan, et al., Appl. Phys. Lett., 2006, 88, 58.

29 P. Y. Yang, H. Wang, X. N. Wang, et al., J. Nanosci. Nanotechnol., 2011, 11, 10804-10808.

30 Q. M. Fu, W. Cao, G. W. Li, et al., Appl. Surf. Sci., 2014, 293, 225-228. 